ANNALES

POLONICI MATHEMATICI

$88.1(2006)$

\title{
On triple curves through a rational triple point of a surface
}

\author{
by M. R. Gonzalez-Dorrego (Madrid)
}

\begin{abstract}
Let $k$ be an algebraically closed field of characteristic 0 . Let $C$ be an irreducible nonsingular curve in $\mathbb{P}^{n}$ such that $3 C=S \cap F$, where $S$ is a hypersurface and $F$ is a surface in $\mathbb{P}^{n}$ and $F$ has rational triple points. We classify the rational triple points through which such a curve $C$ can pass (Theorem 1.8), and give an example (1.12). We only consider reduced and irreducible surfaces.
\end{abstract}

\section{On curves passing through rational triple points of surfaces}

Definition 1.1. Let $F$ be a reduced surface and $P$ a point of $F$. Let $(F, P)$ be a surface singularity (that is, the spectrum of an equicharacteristic local noetherian complete ring of Krull dimension 2, without zero divisors, whose closed point $P$ is singular). Let $\pi: \widetilde{F} \rightarrow F$ be the minimal desingularization of $F$ at $P$. The genus of a normal singularity $P$ is defined to be $\operatorname{dim}_{k}\left(R^{1} \pi_{*} \mathcal{O}_{\widetilde{F}}\right)_{P}$. If the genus is 0 , the singularity is said to be rational. A rational singularity, $P$, such that the multiplicity of the maximal ideal of the local ring $\mathcal{O}_{F, P}$ is 3 , is called a rational triple point. We are going to use configurations of dots and $x\left(\bullet^{2}=-2, x^{2}=-3\right)$ as vertices of the dual graph of the minimal desingularization of the singularity; each vertex corresponds to a curve and each arc to an intersection [1, p. 135]. We list the following singularity types of $P$ :

(1) $X_{i j k}, i, j, k \geq 1 ; i$ denotes the number of dots $\bullet$ to the left of $x, j$ the number of $\bullet$ 's above $x$, and $k$ the number of $\bullet$ 's to the right of $x$.

2000 Mathematics Subject Classification: 14H45, 14J17, 14J25.

Key words and phrases: rational triple singularity, surface singularity, minimal desingularization, maximal cycle, fundamental cycle.

We would like to thank the Department of Mathematics at the University of Toronto for its hospitality during the preparation of this manuscript. 
(2) $Y_{i}, i \geq 1 ; i$ denotes the number of $\bullet$ 's to the left of $x$.

(3) $R_{i j}, i, j \geq 1 ; i$ denotes the number of $\bullet$ 's to the left of $x$ and $j$ the number of $\bullet$ to the right of the vertical edge of the graph.

(4) $T_{i}, i \geq 1 ; i$ denotes the number of $\bullet$ 's to the left of $x$.

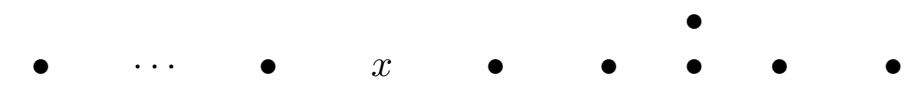

(5) $U_{i j}, i \geq 1, j \geq 2 ; i$ denotes the number of $\bullet$ 's to the left of $x$, and $j$ the number of $\bullet$ 's between $x$ and the vertical edge of the graph.

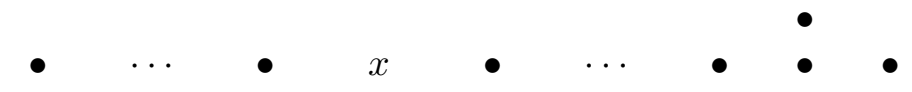

(6) $V_{i}, i \geq 1 ; i$ denotes the number of $\bullet$ 's to the left of the vertical edge of the graph.

(7) $W_{2}$.

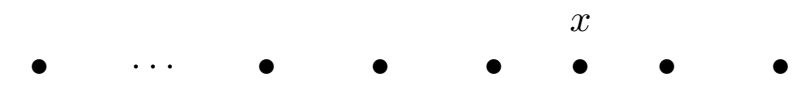




\section{DEFINITION 1.3.}

(1) The maximal cycle is the cycle $Z_{\widetilde{F}}=\sum m_{i} E_{i}$, defined by the divisorial part of $\mathcal{M \mathcal { O } _ { \widetilde { F } }}$, where $\mathcal{M}$ is the maximal ideal $\operatorname{Max} \mathcal{O}_{F, P}$ of $\mathcal{O}_{F, P}$; the $E_{i}$ are the irreducible components of dimension 1 of the exceptional fiber $\pi^{-1}(P)$ and the $m_{i}$ are nonnegative integers. A component $E_{j}$ such that $m_{j}=1$ is called a reduced component of the cycle.

(2) Consider positive cycles $Z=\sum r_{i} E_{i}, r_{i} \geq 0$, such that

$$
\left(Z . E_{i}\right) \leq 0 \quad \text { for all } i \text {. }
$$

The unique componentwise smallest cycle $Z$ satisfying this condition is called the fundamental cycle of $\widetilde{F}$.

Proposition 1.4 (see $[2,1.2])$. Let $(F, P)$ be a complete surface singularity. For any irreducible component $E$ of $\pi^{-1}(P)$, let $\operatorname{ord}_{E}$ denote the divisorial valuation of the function field of $(F, P)$ given by the filtration of $\mathcal{O}_{\widetilde{F}, E}$ by the powers of its maximal ideal. The components $E$ such that

$$
\mathcal{L}_{E}:=\left\{\Gamma \in \mathcal{L} \mid \Phi_{\widetilde{F}}(\Gamma) \in E\right\} \neq \emptyset
$$

are those for which $\operatorname{ord}_{E}\left(\mathcal{M O}_{\widetilde{F}}\right)=1$. The set $\mathcal{L}$ is the disjoint union of the $\mathcal{L}_{E}$.

LEMma 1.5 (see $[2,1.14]$ ). The families of smooth curves on a normal surface singularity are in one-to-one correspondence with the reduced components of the maximal cycle of its minimal desingularization $\pi$.

Note 1.5.1. For a rational surface singularity, the maximal cycle of $\pi$ and the fundamental cycle of its weighted dual graph coincide [1].

COROLlary 1.6. If an irreducible nonsingular curve $C$ passes through a rational singularity $P$ of a surface $F$, then its strict transform must intersect transversally only one reduced component of the fundamental cycle.

Proof. By Lemma 1.5 and Note 1.5.1 the families of nonsingular curves on a rational surface singularity are in one-to-one correspondence with the reduced components of the fundamental cycle of its minimal desingularization. By Proposition 1.4, $C \in \mathcal{L}_{E}$ where $E$ is an irreducible component of $\pi^{-1}(P)$ such that $\operatorname{ord}_{E}\left(\mathcal{M O}_{\widetilde{F}}\right)=1$; thus its strict transform must intersect $E$ transversally, and can intersect no other irreducible exceptional curves because the set of nonsingular curves $C$ on $(F, P)$ whose generic point lies on $\operatorname{Reg}(F), \mathcal{L}$, is a disjoint union of $\mathcal{L}_{E}$ by 1.4 .

1.6.1. Fundamental cycles for rational triple singularities. We exhibit the fundamental cycle for each singularity type. 
(1) Case $X_{i j k}, i, j, k \geq 1$. The fundamental cycle is
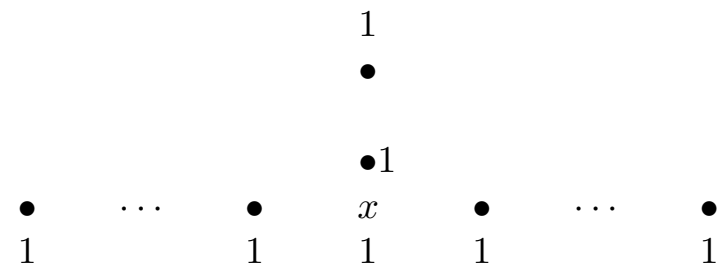

(2) Case $Y_{i}, i \geq 1$. The fundamental cycle is

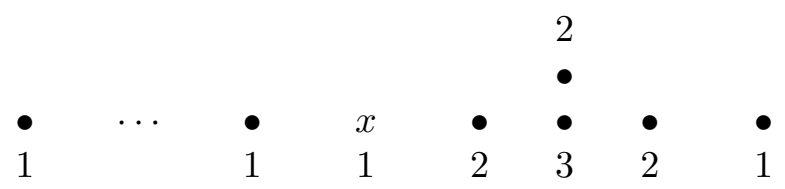

(3) Case $R_{i j}, i, j \geq 1$. The fundamental cycle is

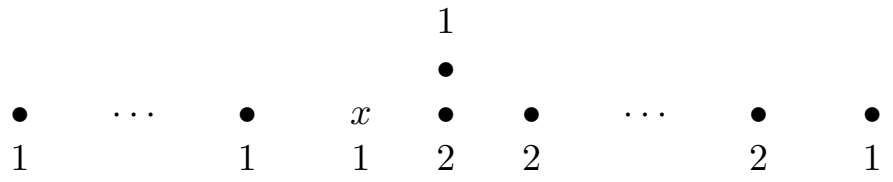

(4) Case $T_{i}, i \geq 1$. The fundamental cycle is

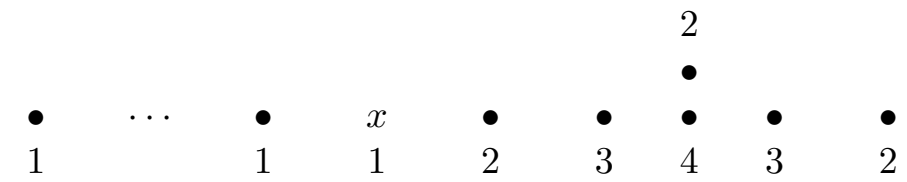

(5) Case $U_{i j}, i \geq 1, j \geq 2$. The fundamental cycle is

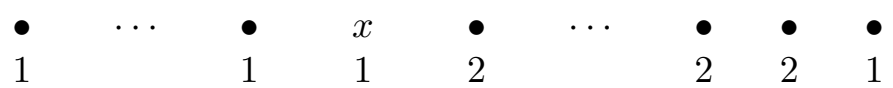

(6) Case $V_{1}$. The fundamental cycle is

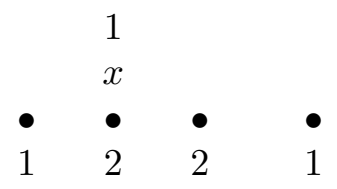

(7) Case $V_{i}, i \geq 2$. The fundamental cycle is

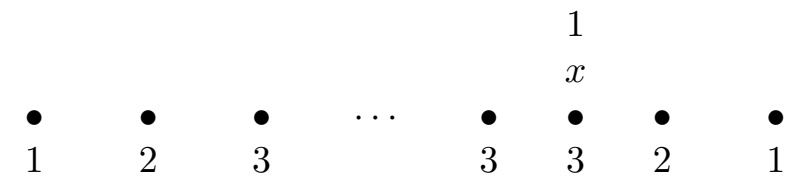


(8) Case $W_{2}$. The fundamental cycle is

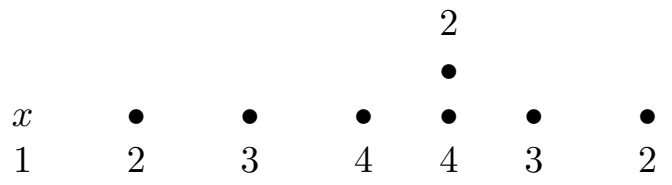

(9) Case $W_{3}$. The fundamental cycle is

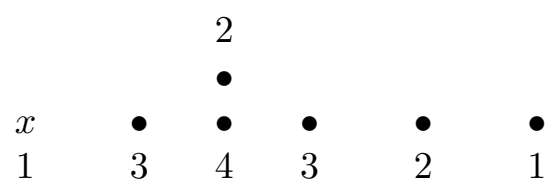

(10) Case $W_{4}$. The fundamental cycle is

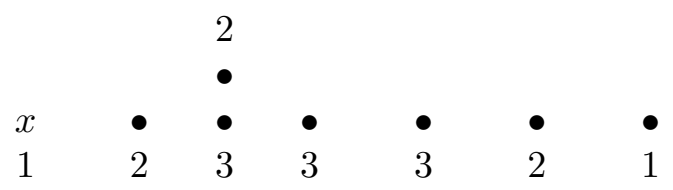

1.7.0. Let $C$ be an irreducible nonsingular curve with $3 C=S \cap F$, where $S$ is a hypersurface and $F$ is a surface with rational triple points. Suppose that $C$ passes through a rational triple point $P$ of $F$. Let $\widetilde{F}$ be the minimal desingularization of $F$ at $P, \pi: \widetilde{F} \rightarrow F$. Let $E_{k}, 1 \leq k \leq n$, be the irreducible components of the exceptional divisor. The total transform $\pi^{*}(3 C)$ equals $\sum_{j=1}^{n} \beta_{j} E_{j}+3 E$, where $E$ is the strict transform of $C, \beta_{j} \in \mathbb{N}$.

Lemma 1.7. Let $E$ and $E_{j}, 1 \leq j \leq n$, be as in 1.7.0. The square of the exceptional cycle of $C$ is

$$
\left(\sum_{j=1}^{n} \beta_{j} E_{j}\right)^{2}=-3 E \cdot\left(\sum_{j=1}^{n} \beta_{j} E_{j}\right)=-3 \beta_{l}
$$

where $l$ is the unique natural number such that $E_{l} \cap E \neq \emptyset$.

Proof. Since $\sum_{j=1}^{n} \beta_{j} E_{j}+3 E$ is a Cartier divisor, it has intersection 0 with $E_{j}$ for all $j$. Thus, $\left(\sum_{j=1}^{n} \beta_{j} E_{j}+3 E\right)\left(\sum_{j=1}^{n} \beta_{j} E_{j}\right)=0$.

THeOREM 1.8. Let $C$ be as in 1.7.0. The square of the exceptional cycle of $C$ is -6 if $C$ passes through one singularity of type $X_{i i i}, i \geq 1$, or of type $R_{11}$, or of type $U_{1 j}, j \geq 2$, or of type $W_{2}$, or of type $W_{3}$. If $C$ passes through one singularity of type $V_{i}, i \in \mathbb{N}$, the square of the exceptional cycle is -9 ; moreover if $i=3 b+2$ for $b \in \mathbb{Z}^{+}$, the square of the exceptional cycle is $-3 b-9$, and if $i=3 a-1, a \in \mathbb{N}$, it is $-3 a-3$. The curve $C$ cannot pass through any other rational triple point of the surface.

Proof. By Corollary 1.6, if an irreducible nonsingular curve $C$ passes through a rational singularity of $F$, then its strict transform must intersect transversally only one reduced component of the fundamental cycle. We 
consider $3 C$ and consider its total transform which must have intersection 0 with each exceptional divisor.

The numbers in the diagrams below under the dots are the multiplicities of the $E_{i}$ 's, i.e. the $\beta_{i}$ 's. The number assigned to the small circle is the multiplicity of $E$ in the cycle $\pi^{*}(3 C)$.

(1) Case $X_{i j k}, i, j, k \geq 1$. Consider its fundamental cycle. Let $a$ be the multiplicity with which $E_{1}$ appears in the total transform.

If we could find, for $a \in \mathbb{N}, b, c \in \mathbb{Z}$, a cycle

$$
(k+1) a-j c
$$

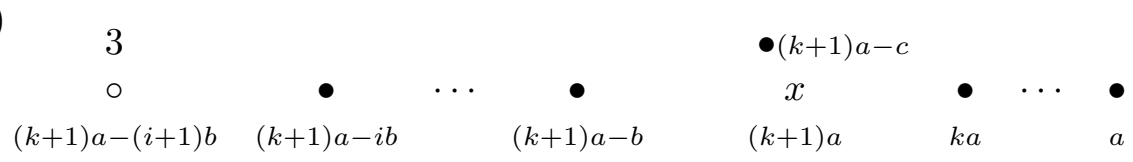

then $C$ would pass through a rational singularity of type $X_{i j k}, i, j, k \geq 1$. Since

$$
\begin{aligned}
& (k+1) a-(i+1) b=3, \\
& (k+1) a-b+k a+(k+1) a-c+(k+1) a(-3)=0,
\end{aligned}
$$

we have $a=\frac{3+(i+1) b}{k+1}, a=-b-c$. On the other hand,

$$
2((k+1) a-j c)=(k+1) a-(j-1) c
$$

implies that $a=\frac{c(j+1)}{k+1}$, so $c>0$. Thus, $b=\frac{c(j+1)-3}{i+1}$. Therefore,

$$
c=\frac{3(k+1)}{(j+1)(i+1)+(i+j+2)(k+1)} \notin \mathbb{Z} .
$$

The cycle (1.8.1) cannot occur.

The cycle

$$
(k+1) a-j c
$$
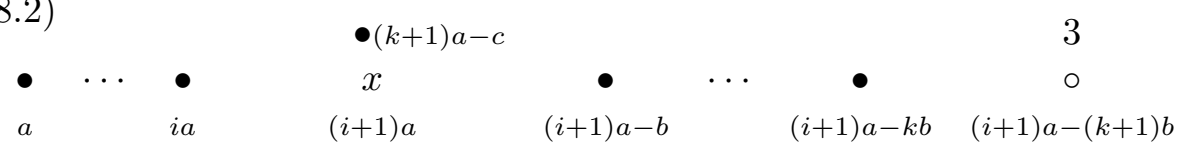

is symmetric to (1.8.1) ( $k$ and $i$ are interchanged). 
Let us see whether we can find, for $a \in \mathbb{N}, b, c \in \mathbb{Z}$, a cycle of the form

$$
\begin{gathered}
(k+1) a-(j+1) c \\
\circ 3 \\
\bullet(k+1) a-j c
\end{gathered}
$$

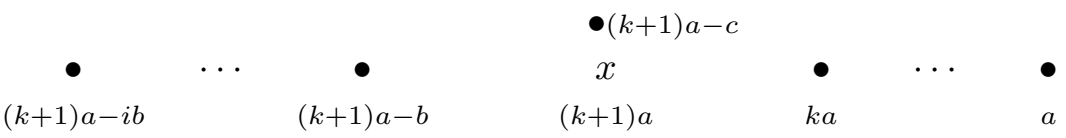

From the equations

$$
\begin{aligned}
& (k+1) a-b+(k+1) a-c+k a+(k+1) a(-3)=0, \\
& (k+1) a-(j+1) c=3,
\end{aligned}
$$

we obtain

$$
a=-b-c, \quad c=-\frac{3+(k+1) b}{k+j+2} .
$$

Since

$$
2((k+1) a-i b)=(k+1) a-(i-1) b,
$$

we get $a=\frac{(i+1) b}{k+1} ;$ so $b>0$. Thus,

$$
b=\frac{3 k+3}{k(i+j+2)+i j+2 i+2 j+3} \notin \mathbb{Z} .
$$

Therefore, the cycle (1.8.3) cannot occur.

Let $l$ be the unique natural number, $1<l<i$, such that $E_{l} \cap E \neq \emptyset$. Let us see whether we can find, for $a \in \mathbb{N}, b, c \in \mathbb{Z}$, a cycle of the form

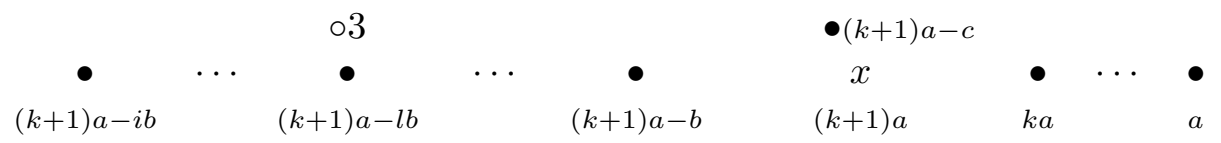

The equation

$$
(k+1) a-(l+1) b+(k+1) a-(l-1) b+3+((k+1) a-l b)(-2)=0
$$

leads to a contradiction. Thus, the cycle (1.8.4) cannot occur. 
Let us see whether we can find, for $a \in \mathbb{N}, b, c \in \mathbb{Z}$, a cycle of the form

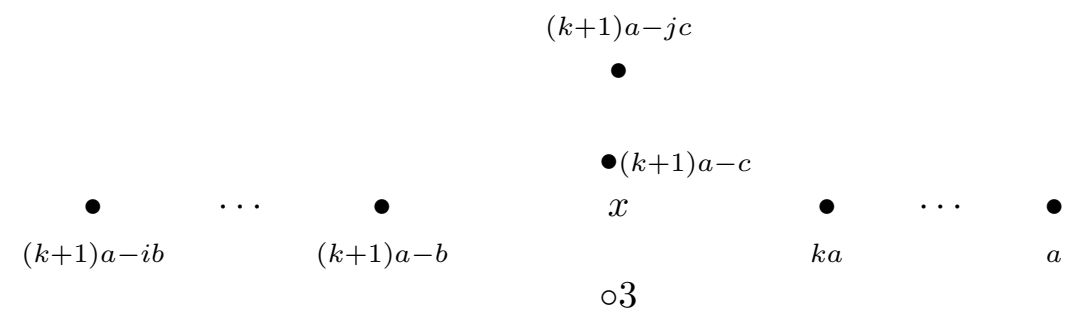

From the equations

$$
\begin{aligned}
& (k+1) a-b+3+(k+1) a-c+k a+(k+1) a(-3)=0, \\
& 2((k+1) a-j c)=(k+1) a-(j-1) c, \\
& 2((k+1) a-i b)=(k+1) a-(i-1) b,
\end{aligned}
$$

we obtain

$$
a=-b-c+3, \quad a=\frac{c(j+1)}{(k+1)}, \quad a=\frac{b(i+1)}{k+1} ;
$$

so, $b>0, c>0$. Since $a=-b-c+3$, we get $b=1$ and $c=1$, which implies that $a=1$. Thus, $i=j=k$; so $C$ can pass through a singularity of type $X_{i i i}$.

We have $\left(\sum_{j=1}^{i} \beta_{j} E_{j}\right)^{2}=-6$. In this case, $\beta_{l}$ of Lemma 1.7 is 2 .

We have shown that $C$ can pass through a singularity of type $X_{i i i}, i \geq 1$, but not through an $X_{i j k}$ singularity with $i \neq j$ or $j \neq k$ or $i \neq k$.

(2) Case $Y_{i}, i \geq 1$. Consider its fundamental cycle. If we could find, for $2 a, 3 a \in \mathbb{N}, b \in \mathbb{Z}, d=6(i+2) a-(3(i+1)+2) b$, a cycle

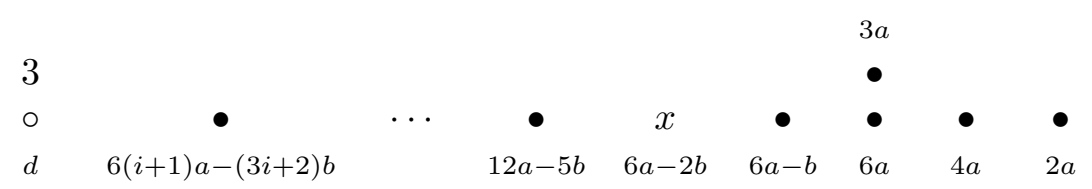

then $C$ would pass through a rational singularity of type $Y_{i}, i \geq 1$. From the equations

$$
6 a-b+4 a+3 a+6 a(-2)=0, \quad 6(i+2) a-(3(i+1)+2) b=3,
$$

we obtain $a=b=\frac{3}{3 i+7} \notin \mathbb{Z}$ for $i \geq 1$.

We consider $3 C$ and its total transform $\pi^{*}(3 C)$ which must have intersection 0 with each exceptional divisor. Let $l$ be the unique natural number, $1<l \leq i$, such that $E_{l} \cap E \neq \emptyset$. Number the exceptional curves $E_{t}$ as 
follows:

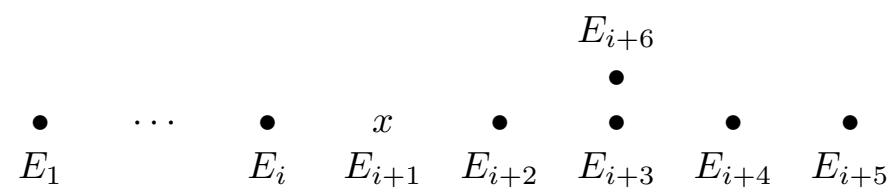

Let $a$ and $b$ denote the respective multiplicities with which $E_{1}$ and $E_{i+5}$ appear in $\pi^{*}(3 C)$. Then the equation $\pi^{*}(3 C) \cdot E_{t}=0$ for all $t$ and the fact that $E$ meets only $E_{l}$ and no other exceptional curve implies that the cycle $\pi^{*}(3 C)$ has the form, for $a \in \mathbb{N}, b \in 2 \mathbb{N}$,

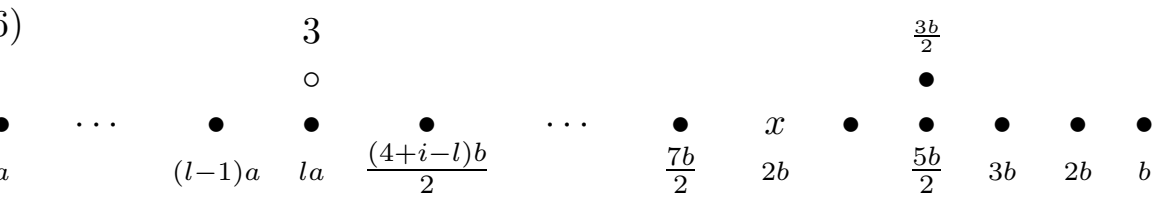

Now, $\pi^{*}(3 C) \cdot E_{l}=0$ reads $2 l a=(l-1) a+3+(4+i-l) b / 2$. Putting this together with the equation $l a=(7+i-l) b / 2$, we obtain $(l+1) a=$ $3+l a-3 b / 2$ or $a=3-3 b / 2$. Since no strictly positive integers $a$ and $b$ can satisfy the last equality, we arrive at a contradiction.

Let us see whether we can find, for $a,(3 i+5) a / 2 \in \mathbb{N}, b \in \mathbb{Z}, d=$ $(3 i+5) a-3 b$, a cycle of the form

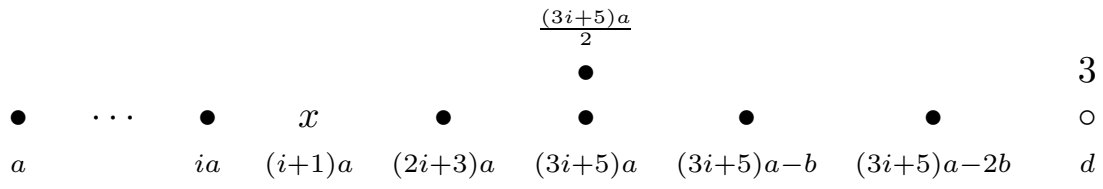

From the equations

$$
\begin{aligned}
& (3 i+5) a-b+(2 i+3) a+\frac{(3 i+5) a}{2}+(3 i+5) a(-2)=0, \\
& (3 i+5) a-3 b=3,
\end{aligned}
$$

we obtain $(i+1) a=2 b, a=\frac{6}{3 i+7} \notin \mathbb{N}$ for $i \geq 1$.

For $2 a, 3 a \in \mathbb{N}, b \in \mathbb{Z}$, consider a cycle

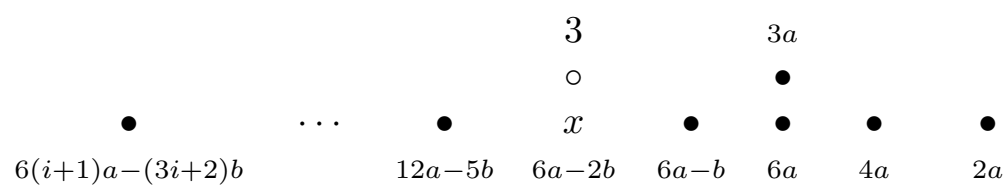

The equation $6 a-b+12 a-5 b+3+(6 a-2 b)(-3)=0$ leads to a contradiction. Thus, $C$ cannot pass through a $Y_{i}$ singularity for $i \geq 1$.

(3) Case $R_{i j}, i, j \geq 1$. Consider its fundamental cycle. If we could find, for $2 a,(j+1) a \in \mathbb{N}, b \in \mathbb{Z}, d=2(i+1)(j+1) a-(2 i+1) b$, a cycle 


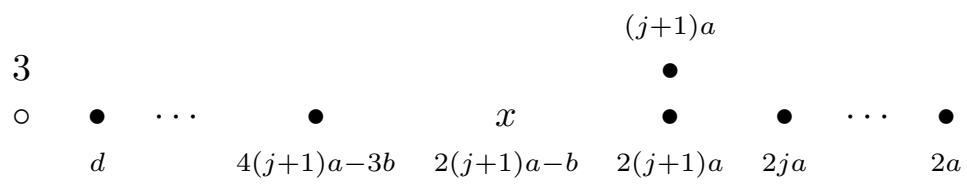

then $C$ would pass through a rational singularity of type $R_{i j}, i, j \geq 1$. From the equations

$$
\begin{aligned}
& 2(j+1) a-b+(j+1) a+2 j a+2(j+1) a(-2)=0, \\
& 2(i+2)(j+1) a-(2(i+1)+1) b=3,
\end{aligned}
$$

we obtain $(j-1) a=b, a=\frac{3}{4 i+j+7}, 2 a \notin \mathbb{N}$ for $i, j \geq 1$.

Let $2 a \in \mathbb{N}, d=2(2 i+3) a-(j+1) b$. If we could find a cycle

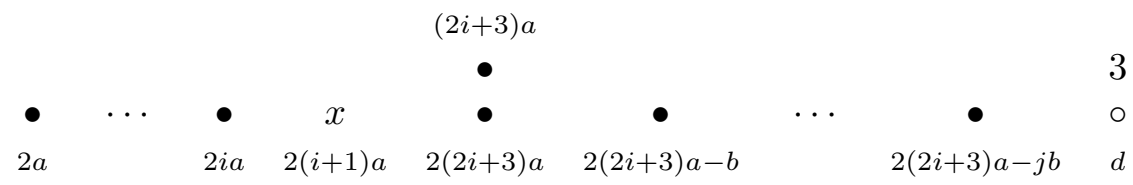

then $C$ would pass through a rational singularity of type $R_{i j}, i, j \geq 1$. From the equations

$$
\begin{aligned}
& 2(i+1) a+2(2 i+3) a-b+(2 i+3) a=4(2 i+3) a, \\
& 2(2 i+3) a-(j+1) b=3,
\end{aligned}
$$

we obtain $a=-b, a=\frac{3}{4 i+j+7}, 2 a \notin \mathbb{N}$ for $i, j \geq 1$.

Let us see whether we can find, for $2 a \in \mathbb{N}, b, c \in \mathbb{Z}, d=2(i+1)(j+1) a$ $-(2 i+1) b$, a cycle of the form

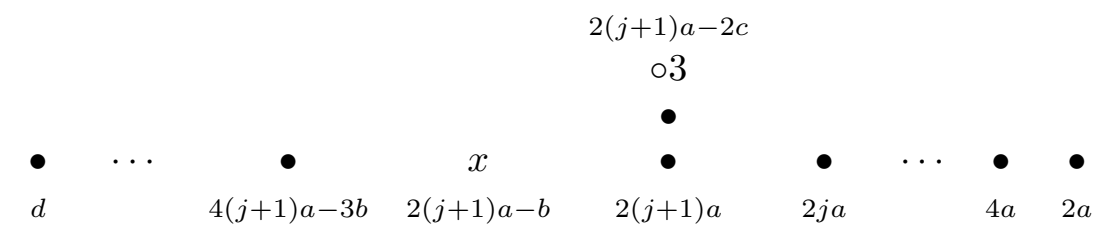

From the equations

$$
\begin{aligned}
& 2(j+1) a-2 c=3, \\
& 2(j+1) a-b+2(j+1) a-c+2 j a+2(j+1) a(-2)=0,
\end{aligned}
$$

we obtain

$$
c=\frac{2(j+1) a-3}{2}, \quad b=\frac{2(j-1) a+3}{2} .
$$

Since

$$
2(2(i+1)(j+1) a-(2 i+1) b)=2 i(j+1) a-(2 i-1) b,
$$

we get $a=\frac{3}{8 i+6 j+10}$; so $2 a \notin \mathbb{N}$ for $i, j \geq 1$.

Let $l$ be the unique natural number, $1<l \leq i$, such that there is no cycle with $E_{l} \cap E \neq \emptyset$ (see (1.8.6)). 
Consider, for $a \in \mathbb{N}, b, c \in \mathbb{Z}, \frac{(i+1) a-b}{2} \in \mathbb{N}$, a cycle

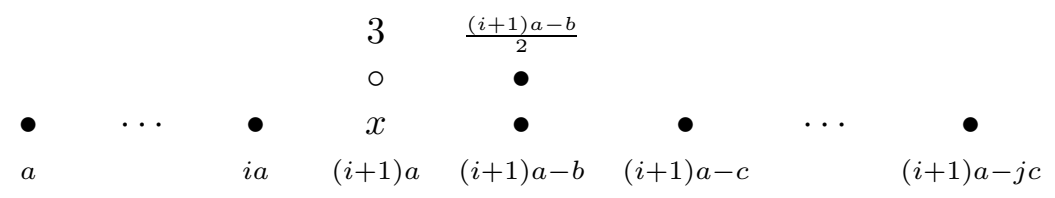

From the equation

$$
(i+1) a-b+3+i a+(i+1) a(-3)=0,
$$

we obtain $b=3-(i+2) a$. From

$$
(i+1) a+\frac{(i+1) a-b}{2}+(i+1) a-c+((i+1) a-b)(-2)=0,
$$

we obtain $\frac{9-(2 i+5) a}{2}=c$. On the other hand,

$$
2((i+1) a-j c)=(i+1) a-(j-1) c,
$$

so $c=\frac{9(i+1)}{2(i+1)(j+2)+3(j+1)} \geq 1 ;$ since $a \in \mathbb{N}$ and $a=\frac{9-2 c}{2 i+5}$, we get $c=1$. Consequently, $i=1, a=1, b=0, j=1$.

We have $\left(\sum_{j=1}^{5} \beta_{j} E_{j}\right)^{2}=\left(E_{1}+2 E_{2}+2 E_{3}+E_{4}+E_{5}\right)^{2}=-6$.

In this case, $\beta_{l}$ of Lemma 1.7 is 2 .

Hence, $C$ can pass through a rational singularity of type $R_{11}$, but not through a rational singularity of type $R_{i j}$ with $i$ or $j>1$.

(4) Case $T_{i}, i \geq 1$. Consider its fundamental cycle. If we could find, for $2 a, 3 a \in \mathbb{N}, b \in \mathbb{Z}, d=(i+1) 6 a-(5 i+2) b$, a cycle

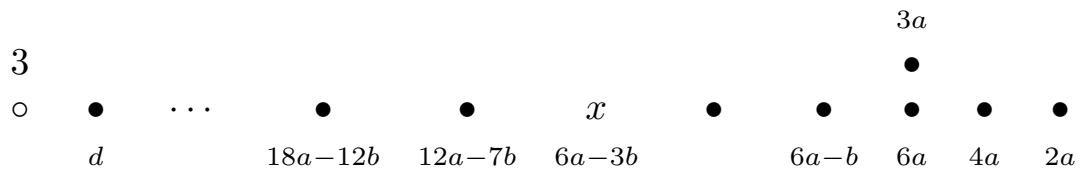

then $C$ would pass through a rational singularity of type $T_{i}, i \geq 1$. From the equations

$$
6 a-b+4 a+3 a+6 a(-2)=0, \quad(i+2) 6 a-(5 i+7) b=3,
$$

we see that $a=b$ and $i=-2$, which is absurd.

Let $l$ be the unique natural number, $1<l \leq i$, such that there is no cycle such that $E_{l} \cap E \neq \emptyset$.

Consider, for $a \in \mathbb{N}, b, c \in \mathbb{Z}, \frac{(i+1) a-3 b}{2} \in \mathbb{N}, d=(i+1) a-2 c$, a cycle

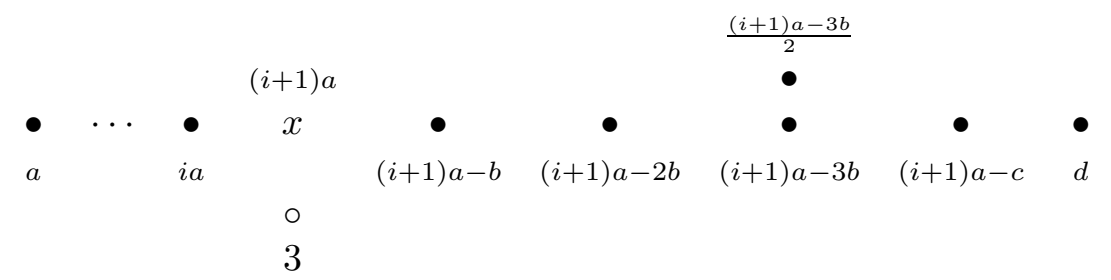


From the equations

$$
\begin{aligned}
& i a+(i+1) a-b+3+(i+1) a(-3)=0, \\
& (i+1) a-2 b+\frac{(i+1) a-3 b}{2}+(i+1) a-c+((i+1) a-3 b)(-2)=0,
\end{aligned}
$$

we obtain $b=3-(i+2) a$ and $c=\frac{15-(4 i+9) a}{2}$. On the other hand,

$$
2((i+1) a-2 c)=(i+1) a-c ;
$$

so $a=\frac{45}{14 i+29} \notin \mathbb{N}$ for $i \geq 1$.

Hence, no $C$ can pass through a $T_{i}$ singularity for $i \geq 1$.

(5) Case $U_{i j}, i \geq 1, j \geq 2$. Consider its fundamental cycle. If we could find, for $2 a \in \mathbb{N}, b \in \mathbb{Z}, d=4(i+1) a-((i+1) j+(2 i+1)) b$, a cycle

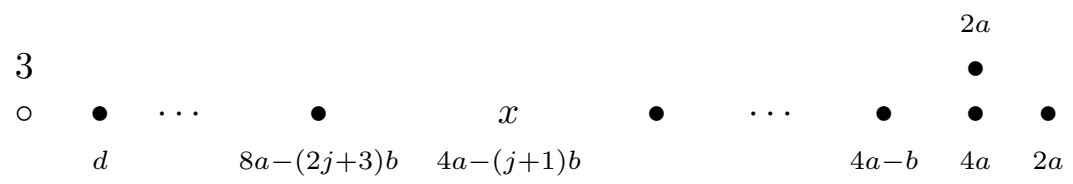

then $C$ would pass through a rational singularity of type $U_{i j}, i \geq 1, j \geq 2$. From the equations

$$
\begin{aligned}
& 4 a-b+2 a+2 a+4 a(-2)=0, \\
& 4(i+2) a-((i+2) j+(2 i+3)) b=3,
\end{aligned}
$$

we obtain $b=0, a=\frac{3}{4(i+2)}, 2 a \notin \mathbb{N}$ for $i \geq 1$.

As in (1.8.6), we cannot find a cycle

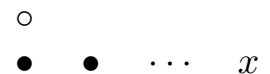

Let us see whether we can find, for $a, \frac{((j+2) i+(2 j+3)) a}{2} \in \mathbb{N}, b \in \mathbb{Z}, d=$ $((j+2) i+(2 j+3)) a-b$, a cycle

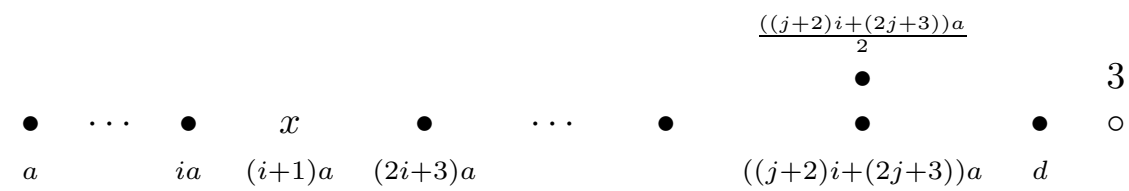

From the equations

$$
\begin{gathered}
\left.((j+1) i+(2 j+1)) a+\frac{((j+2) i+}{2}(2 j+3)\right) a \\
+((j+2) i+(2 j+3)) a-b \\
+((j+2) i+(2 j+3)) a(-2)=0, \\
((j+2) i+(2 j+3)) a-2 b=3,
\end{gathered}
$$

we obtain $a=\frac{3}{2(i+2)} \notin \mathbb{N}$ for $i \geq 1$. 
Consider, for $2 a \in \mathbb{N}, b, c \in \mathbb{Z}, d=4(i+1) a-((i+1) j+(2 i+1)) b$ a cycle

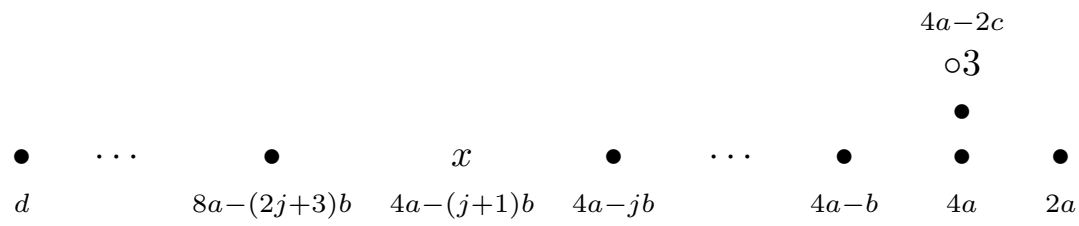

From the equations

$$
4 a-b+4 a-c+2 a+4 a(-2)=0, \quad 4 a-2 c=3,
$$

we obtain $b+c=2 a, c=\frac{4 a-3}{2}$; so $b=\frac{3}{2} \notin \mathbb{Z}$.

Consider, for $a \in \mathbb{N}, b, c \in \mathbb{Z}, \frac{(i+1) a-(j+1) b}{2} \in \mathbb{N}$, a cycle

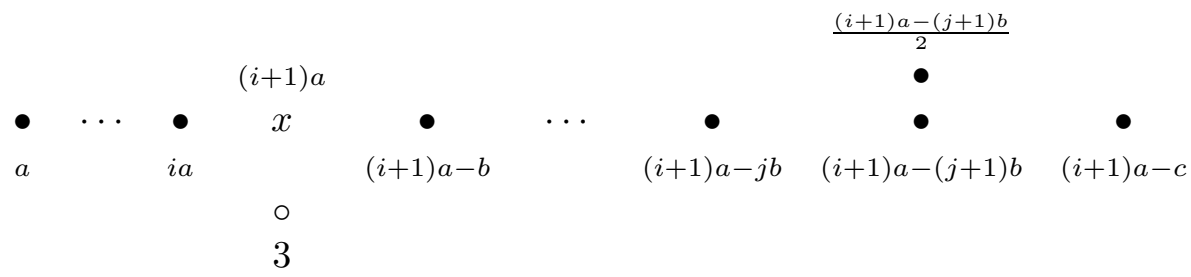

From the equations

$$
\begin{gathered}
i a+(i+1) a-b+3+(i+1) a(-3)=0, \\
(i+1) a-j b+\frac{(i+1) a-(j+1) b}{2}+(i+1) a-c+((i+1) a-(j+1) b)(-2)=0,
\end{gathered}
$$

we obtain

$$
b=3-(i+2) a, \quad c=\frac{(i+1) a+(j+3) b}{2},
$$

so $c=\frac{9+3 j-((i+2)(j+2)+1) a}{2}$. Since

$$
2((i+1) a-c)=(i+1) a-(j+1) b,
$$

we get $c-2 b=c$, so $b=0$; thus $a=\frac{3}{i+2} \in \mathbb{N}$ implies that $i=1$ and $a=1$; so $c=1$ for $j \geq 1$.

Hence, $C$ can pass through a rational singularity of type $U_{1 j}$ for $j \geq 2$, but there are no $C$ passing through an $U_{i j}$ singularity for $i>1, j \geq 2$.

(6) Case $V_{i}, i \geq 1$. Consider its fundamental cycle. For $2 a \in \mathbb{N}, b \in \mathbb{Z}$, consider a cycle

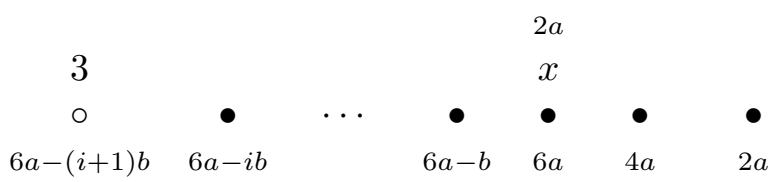


From the equations

$$
\begin{aligned}
& 6 a-b+2 a+4 a+6 a(-2)=0, \\
& 6 a-(i+1) b=3,
\end{aligned}
$$

we obtain $b=0,2 a=1$; we have the cycle

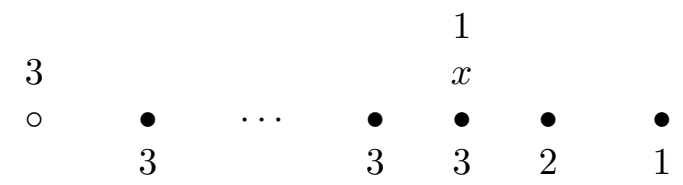

For $i \in \mathbb{N}$, the singularity $V_{i}$ can occur.

For $a \in \mathbb{N}, b \in \mathbb{Z}, \frac{(i+1) a}{3} \in \mathbb{N}$, consider a cycle

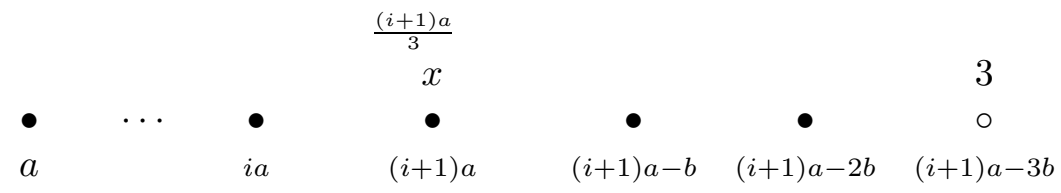

Since

$$
\begin{aligned}
& i a+\frac{(i+1) a}{3}+(i+1) a-b+(-2)(i+1) a=0, \\
& (i+1) a-3 b=3,
\end{aligned}
$$

we obtain $a=1$ and $b=\frac{i-2}{3}$. In this case, the total transform of $3 C$, for $b \in \mathbb{Z}^{+}$, is

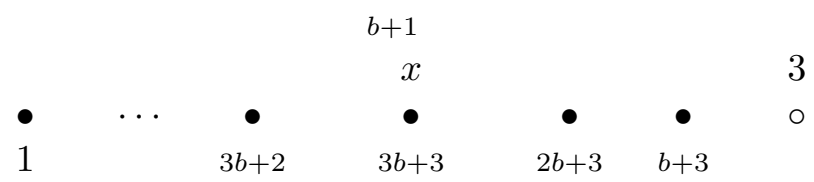

For $b \in \mathbb{Z}^{+}$, the singularity $V_{3 b+2}$ can occur.

For $a \in \mathbb{N}, b, c \in \mathbb{Z}$, consider a cycle

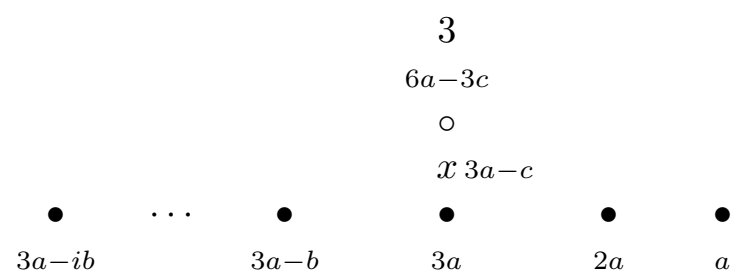

Since $6 a-3 c=3$, we have $c=2 a-1$. From the equation

$$
3 a-b+3 a-c+2 a+3 a(-2)=0,
$$

we obtain $2 a=b+c$; so $b=1$. From $2(3 a-i b)=3 a-(i-1) b$, we get $a=\frac{i+1}{3}$. For $a \geq 1$, the singularity $V_{3 a-1}$ can occur. 
In this case, the total transform of $3 C$ is

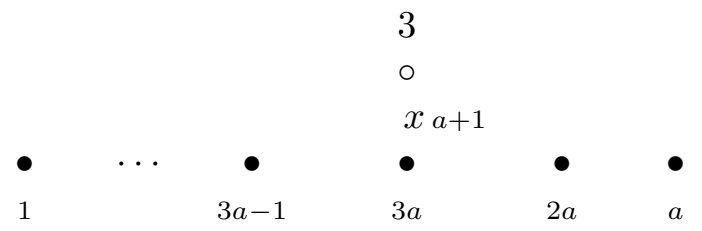

So $C$ can pass through a rational singularity of type $V_{3 a-1}, a \geq 1$.

(7) $C$ ase $W_{2}$. Consider its fundamental cycle. If we could find, for $2 a, 3 a$ $\in \mathbb{N}, b \in \mathbb{Z}$, a cycle

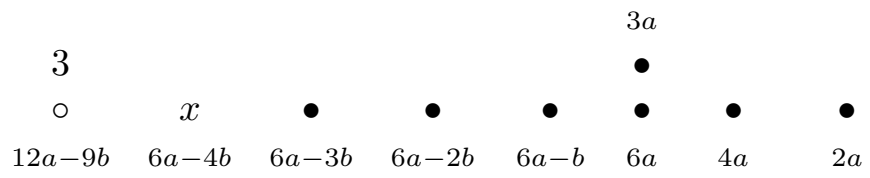

then $C$ would pass through a rational singularity of type $W_{2}$. Let us see if it is possible to find $a$ and $b$ satisfying the equations $12 a-9 b=3$ and $6 a-b+4 a+3 a+6 a(-2)=0$. From the second equation we obtain $a=b$. Thus $a=1$.

We have $\left(\sum_{j=1}^{8} \beta_{j} E_{j}\right)^{2}=-6$. In this case, $\beta_{l}$ of Lemma 1.7 is 2 .

Hence, $C$ can pass through a rational singularity of type $W_{2}$.

(8) Case $W_{3}$. Consider its fundamental cycle. If we could find, for $2 a \in$ $\mathbb{N}, b \in \mathbb{Z}$, a cycle

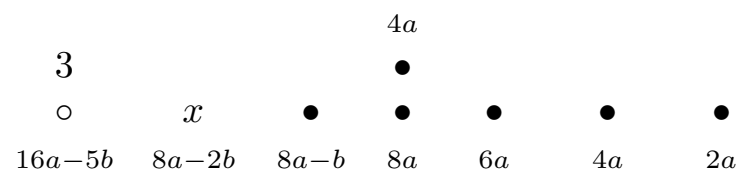

then $C$ would pass through a rational singularity of type $W_{3}$. From the equations

$$
8 a-b+6 a+4 a+8 a(-2)=0, \quad 16 a-5 b=3,
$$

we obtain $2 a=b$ and $a=\frac{1}{2}$, so $2 a=1$.

We have $\left(\sum_{j=1}^{7} \beta_{j} E_{j}\right)^{2}=-6$. In this case, $\beta_{l}$ of Lemma 1.7 is 2 .

Hence, $C$ can pass through a rational singularity of type $W_{3}$.

Let us see whether we can find, for $2 a, 5 a \in \mathbb{N}, b \in \mathbb{Z}$, a cycle

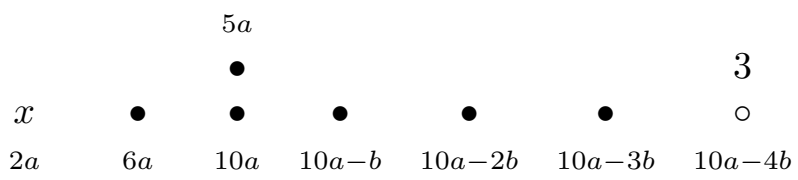

From the equations

$$
6 a+5 a+10 a-b+10 a(-2)=0, \quad 10 a-4 b=3,
$$


we obtain $a=b=\frac{1}{2}$, so $2 a=1$ but $5 a \notin \mathbb{N}$. Thus, this possibility cannot occur.

(9) Case $W_{4}$. If $C$ passed through a rational singularity of type $W_{4}$, we would be able to find, for $2 a, 5 a \in \mathbb{N}, b \in \mathbb{Z}$, a cycle

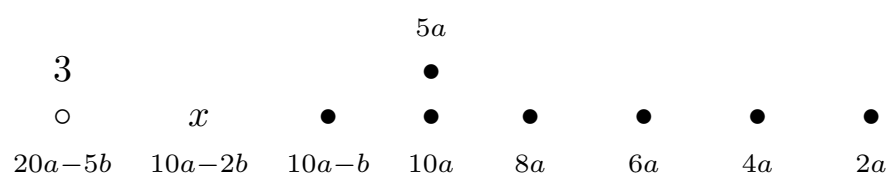

From the equations

$$
10 a-b+8 a+5 a+10 a(-2)=0, \quad 20 a-5 b=3,
$$

we obtain $3 a=b, a=\frac{3}{5}, b \notin \mathbb{Z}$.

Let us see whether we can find, for $2 a, 5 a \in \mathbb{N}, b \in \mathbb{Z}$, a cycle

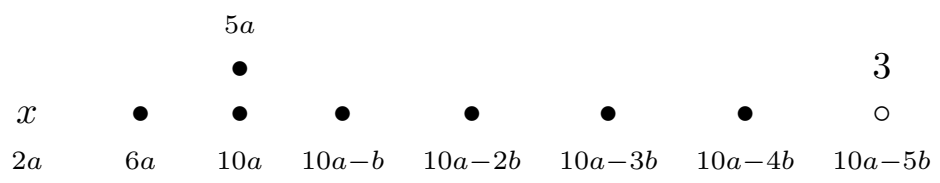

From the equations

$$
6 a+5 a+10 a-b+10 a(-2)=0, \quad 10 a-5 b=3,
$$

we obtain $a=b=\frac{3}{5}, b \notin \mathbb{Z}$. Thus, $C$ cannot pass through a $W_{4}$ singularity.

Definition 1.9. Let $R$ be a regular 2-dimensional local noetherian ring. A sandwiched singularity is the singularity of a blowing-up of Spec $R$ along a complete ideal [4, p. 432].

Note 1.10. A normal surface singularity is minimal if and only if it is rational with reduced fundamental cycle [3, 4.4.10]

Proposition 1.11 ([4, Proposition 2.4]). Every minimal singularity is sandwiched.

EXAMPLE 1.12. A singularity of type $X_{111}$ is a sandwiched singularity because it is minimal (1.11). It is minimal because it is a rational singularity with reduced fundamental cycle (1.10). We consider the blowing-up of Spec $k[x, y]$ along the complete ideal $\left(y x^{4}, x^{6}, y^{2}+y x^{2}\right)$. It has a unique singularity with local coordinates $\left(x, y, t^{\prime}, u^{\prime}\right)$, where

$$
t^{\prime}=\frac{y x^{4}}{y^{2}+y x^{2}}, \quad u^{\prime}=\frac{x^{6}}{y^{2}+y x^{2}} .
$$

This blowing-up is a surface $F$ defined in $\mathbb{A}_{k}^{4}$ by the relations

$$
y\left(t^{\prime}+u^{\prime}\right)=x^{4}, \quad u^{\prime} y=t^{\prime} x^{2}, \quad u^{\prime} x^{2}=t^{\prime 2}+t^{\prime} u^{\prime} .
$$


Let $I$ be the ideal generated in $k\left[x, y, t^{\prime}, u^{\prime}\right]$ by the relations above. Then $k\left[x, y, t^{\prime}, u^{\prime}\right] / I$ is a free module over $k\left[y, u^{\prime}\right]$ generated by $\left(1, x, x^{2}, x^{3}, t^{\prime}, t^{\prime} x\right)$; it is the affine coordinate ring of the surface $F$ with a rational triple point $X_{111}$ at the origin. In $k\left[x, y, t^{\prime}, u^{\prime}\right]$ we consider the ideal $J$ generated by the irreducible polynomial $f\left(x, y, t^{\prime}, u^{\prime}\right)=7 t^{\prime}-u^{\prime}+y-4 x^{2}$. This polynomial is obtained as follows. Let us look at the dual graph (1.8.6). Then $f\left(x, y, t^{\prime}, u^{\prime}\right)$ equals $\frac{\left(y-x^{2}\right)^{3}}{y\left(y+x^{2}\right)}$ modulo $I$. The quotient $k\left[x, y, t^{\prime}, u^{\prime}\right] / J$ is the affine coordinate ring of a hypersurface $S$. After projectivization, the intersection of $S$ and $F$ is a multiplicity-three structure on a curve $C$ passing through the singularity $X_{111}$ of $F$.

\section{References}

[1] M. Artin, On isolated rational singularities of surfaces, Amer. J. Math. 88 (1966), 129-136.

[2] G. González-Sprinberg and M. Lejeune-Jalabert, Families of smooth curves on singularities and wedges, Ann. Polon. Math. 67 (1997), 179-190.

[3] J. Kollár, Toward moduli of singular varieties, thesis, Brandeis Univ., 1983.

[4] M. Spivakovsky, Sandwiched singularities and desingularizations of surfaces by normalized Nash-transformations, Ann. of Math. 131 (1990), 411-491.

Departamento de Matemáticas

Universidad Autónoma de Madrid

28049 Madrid, Spain

E-mail: mrosario.gonzalez@uam.es

Received 26.11.2003

and in final form 27.10.2005 